\title{
ON THE SEPARABILITY OF SIGNAL AND INTERFERENCE-PLUS-NOISE SUBSPACES IN BLIND PILOT DECONTAMINATION
}

\author{
Julia Vinogradova, Emil Björnson, and Erik G. Larsson \\ Department of Electrical Engineering (ISY) \\ Linköping University, Sweden
}

\begin{abstract}
Consider a multicell multiuser MIMO (multiple-input multipleoutput) system with a very large number of antennas at each base station (BS). The number of users in each cell is assumed to be fixed as the number of BS antennas grows large. Under certain conditions on the powers of the transmitting users, the signal eigenvalue spectrum is asymptotically separated from the interference-plusnoise spectrum as the number of BS antennas grows large. As it was observed in [1], this phenomenon allows to mitigate the pilot contamination problem. We provide the power limits for each user in the cell of interest above which such a separation occurs asymptotically. Unlike the approximative methods used in [1], we obtain these power limits by making use of the exact asymptotic characterizations of the interference-plus-noise spectrum. The results are based on the theory of small rank perturbations of large dimensional random matrices.
\end{abstract}

Index Terms - Multicell multiuser MIMO, pilot contamination, random matrix theory, spiked models

\section{INTRODUCTION}

Massive MIMO is a promising technique to improve the spectral efficiency of wireless communication systems. The key idea is to let a multitude of users be active simultaneously and separate their signals in spatial domain by equipping the BS with a large number of antennas. Since this separation requires the BS to know spatial channel characteristics of each desired user, channel estimation is one of the main challenges in multicell massive MIMO systems. Due to the limited resources on fading channels, the use of orthogonal pilot sequences across all cells is unfeasible and the same pilots need to be reused in at least a fraction of the cells. Therefore, the channel estimates obtained in the cell of interest are contaminated by signals transmitted by users in the other cells. This problem is referred to as pilot contamination. The corresponding loss in throughput was analyzed in the early works [2] and [3], and the contamination is one of the limiting factors when deploying very large BS arrays. The pilot contamination effect can be suppressed by allocating the pilot sequences in a judicious way. Variations in the spatial correlation between users can be exploited to identify users that cause less pilot contamination to each other $[4,5]$. Alternatively, one can reuse the same pilot sequences in only $1 / f$ of the cells, which reduces the contamination at the cost of having $f$ times more pilots $[4,6]$. Note that only the pilot structure is optimized and utilized for channel estimation in $[4,5,6]$.

Blind and semi-blind estimators were used in $[1,7,8,9]$ to exploit also the payload data for channel estimation. These methods can be used to separate desired signals from interfering signals and noise, if the power levels are sufficiently different. The separability is key in these schemes and was studied in [1], where a blind pilot decontamination method based on the theory of large dimensional random matrices was proposed. Depending on the system parameters, as the dimensions of the received signal matrix grow large, the spectrum of the sample covariance matrix asymptotically decomposes into two disjoint parts corresponding to the interference-plus-noise eigenvalue bulk and the signal eigenvalues. The main idea of [1] was to find an approximated characterization of the limiting supports of the interference-plus-noise spectrum from one side and of the signal spectrum from the other. Then, a bound on the power difference between signal and interference was determined in order for these two bulk to not overlap. However, the method in [1] has two main limitations. First, due to the approximations, the final bound does not depend on the noise variance, so the bound becomes inaccurate in the low signal-to-noise ratio (SNR) regime. The second limitation comes from the approximation based on the assumption that the number of interfering signals and signals of interest are both very small in comparison to the number of BS antennas, making it inapplicable to the scenarios where the number of cells is considered to be very large.

We derive a new asymptotic condition on the signal and interference-plus-noise spectrum separability. The results are obtained by using recent results from the theory of small rank perturbations of large dimensional random matrices. We only need to assume that the number of signals of interest is much smaller than the number of receive antennas at the BS. The results rely on the exact asymptotic analysis of the interference-plus-noise spectrum. The new separability condition provides accurate results also in situations where the approximation from [1] are inaccurate.

Notations: The superscript $(\cdot)^{\mathrm{H}}$ is the Hermitian transpose of a matrix. We denote by $\stackrel{\text { a.s. }}{\longrightarrow}$ the almost sure (a.s.) convergence. The symbol $\Im(z)$ stands for the imaginary part of $z$. We denote by $\mathcal{C N}\left(a, \sigma^{2}\right)$ the complex circular Gaussian distribution with mean $a$ and variance $\sigma^{2}$.

\section{SYSTEM MODEL}

Consider a multicell multiuser MIMO system with a cell of interest and $L$ neighboring interfering cells. Each cell contains one BS equipped with $M$ antennas and $K$ single-antenna users. Consider the uplink (reverse link) transmission where the BS of interest receives signals from all users in all cells. Let $\tau$ be the length of the coherence interval in samples. The $M \times 1$ received vector at time $t=1, \ldots, \tau$ is given by

$$
\mathbf{y}_{t}=\mathbf{H} \mathbf{x}_{t}+\sum_{l=1}^{L} \mathbf{H}^{l} \mathbf{x}_{t}^{l}+\mathbf{w}_{t}
$$


where $\mathbf{x}_{t} \in \mathbb{C}^{K \times 1}$ is the transmitted data vector with independent entries with zero mean and covariance matrix $\mathbf{P}=\operatorname{diag}\left(P_{1}, \ldots, P_{K}\right)$ with decreasingly ordered elements $P_{1} \geq \ldots \geq P_{K}$ representing the powers of the transmitted signals in the home cell ${ }^{1} ; \mathbf{H} \in \mathbb{C}^{M \times K}$ is the channel matrix between the BS and the $K$ users with independent identically distributed (i.i.d.) entries with zero mean and unit variance; $\mathbf{x}_{t}^{l} \in \mathbb{C}^{K \times 1}$ is the interfering data vector in the $l$ th interfering cell with independent $\mathcal{C N}(0, I)$ entries $^{2} ; \mathbf{H}^{l} \in \mathbb{C}^{M \times K}$ is the channel matrix between the BS and the users of the cell $l$ with entries $\left[\mathbf{H}^{l}\right]_{m, k=1}^{M, K} \sim \mathcal{C N}(0,1) ; \mathbf{w}_{t} \in \mathbb{C}^{M \times 1}$ is the additive noise vector with independent $\mathcal{C N}\left(0, \sigma^{2}\right)$ entries. Defining $\widetilde{\mathbf{x}}_{t}=\left[\left(\mathbf{x}_{t}^{1}\right)^{\mathrm{H}}, \ldots,\left(\mathbf{x}_{t}^{L}\right)^{\mathrm{H}}\right]^{\mathrm{H}} \in \mathbb{C}^{L K \times 1}$ and concatenating $\tau$ successive samples of the received vectors given by (1), we obtain

$$
\mathbf{Y}=\mathbf{H} \mathbf{P}^{1 / 2} \mathbf{X}+\mathbf{H}_{I} \mathbf{X}_{I}+\mathbf{W}
$$

where $\mathbf{Y}=\left[\mathbf{y}_{1}, \ldots, \mathbf{y}_{\tau}\right] \in \mathbb{C}^{M \times \tau}$, the matrix $\mathbf{X} \in \mathbb{C}^{K \times \tau}$ has i.i.d. entries, $\mathbf{X}_{I}=\left[\widetilde{\mathbf{x}}_{1}, \ldots, \widetilde{\mathbf{x}}_{\tau}\right] \in \mathbb{C}^{L K \times \tau}$ with $\left[\mathbf{X}_{I}\right]_{k, t=1}^{K, \tau} \sim \mathcal{C N}(0, I)$, $\mathbf{H}_{I}=\left[\mathbf{H}^{1} \mathbf{P}^{1}, \ldots, \mathbf{H}^{L} \mathbf{P}^{L}\right] \in \mathbb{C}^{M \times L K}$ with $\left[\mathbf{H}_{I}\right]_{m, l=1}^{M, L K} \sim$ $\mathcal{C N}(0,1)$, and $\mathbf{W}=\left[\mathbf{w}_{1}, \ldots, \mathbf{w}_{\tau}\right] \in \mathbb{C}^{M \times \tau}$ with $[\mathbf{W}]_{m, t=1}^{M, \tau} \sim$ $\mathcal{C N}(0,1)$.

We assume in the following that $M$ and $\tau$ are large ${ }^{3}$ and of the same order of magnitude and define the asymptotic regime by $M \rightarrow$ $\infty$, such that $M / \tau \rightarrow c \in(0, \infty)$. $K$ is assumed to be fixed as $M \rightarrow \infty$. We define also $\tilde{c}$ such that $M /(L K) \rightarrow \tilde{c} \in(0, \infty)$. Note that the definition of $\tilde{c}$ is more general than the assumption made in [1] where $L K$ is implicitly assumed to be very small as compared to $M$. The results of this paper are based on asymptotic spectral analysis of the sample covariance matrix defined by $\widehat{\mathbf{R}} \triangleq \frac{1}{\tau} \mathbf{Y} \mathbf{Y}^{\mathrm{H}}$.

Write now the model (2) as $\mathbf{Y}=\mathbf{A}+\mathbf{Z}$ where $\mathbf{A}=\mathbf{H} \mathbf{P}^{1 / 2} \mathbf{X}$ is of small rank $K$ with probability one and whose singular values converge to $\sqrt{\tau M P_{1}}, \ldots, \sqrt{\tau M P_{K}}$ as $M \rightarrow \infty$ and $\mathbf{Z}=\mathbf{H}_{I} \mathbf{X}_{I}+$ $\mathbf{W}$ has a rank equal to $\min (M, \tau)$ with probability one. In large dimensional random matrix theory, this model corresponds to a socalled "spiked models" (see, e.g., [10]) where $\mathbf{Z}$ is perturbed by the small rank matrix $\mathbf{A}$. In order to understand the main idea, consider first the interference free case with $\mathbf{Y}=\mathbf{A}+\mathbf{W}$ where the matrix $\mathbf{W}$ corresponds to the noise only part. As $M \rightarrow \infty$, the spectrum of $\widehat{\mathbf{R}}$ converges to the Marčenko-Pastur (MP) law [11] and is composed of one bulk of eigenvalues. However, under some conditions on $\mathbf{P}$, up to $K$ isolated eigenvalues referring to the presence of the signal matrix $\mathbf{A}$ can be found on the right side of the support of the MP law. More precisely, if the smallest power satisfies the condition $P_{K}>\sigma^{2} \sqrt{c} / M$ [10], then all signal eigenvalues are asymptotically separated from the noise eigenvalues as $M \rightarrow \infty$. That is illustrated in Figure 1 where the histogram is plotted for one realization of $\mathbf{Y}$ and where both signal powers satisfy the separability condition.

We come back now to the model $\mathbf{Y}=\mathbf{A}+\mathbf{Z}$. As the interference-plus-noise covariance matrix $\mathbf{Z}$ has non i.i.d. entries, the spectrum of $\widehat{\mathbf{R}}$ does not converge to the MP law and the condition of separability is not the same as above. Asymptotically, the spectrum of $\widehat{\mathbf{R}}$ may consist of one or two bulks (depending on the interference-to-noise ratio, $M, c$, and $\tilde{c}$ ) representing noise and interference and, as above, of up to $K$ isolated eigenvalues corre-

\footnotetext{
${ }^{1}$ Pathloss differences within the home cell can be absorbed into $\mathbf{P}$.

${ }^{2}$ The equal interference variance from all neighboring cells represents a worst case scenario. Ongoing work considers the case where this variance is different in different cells.

${ }^{3}$ Note that practical values of $M$ and $\tau$ are at the order of hundreds or thousands.
}

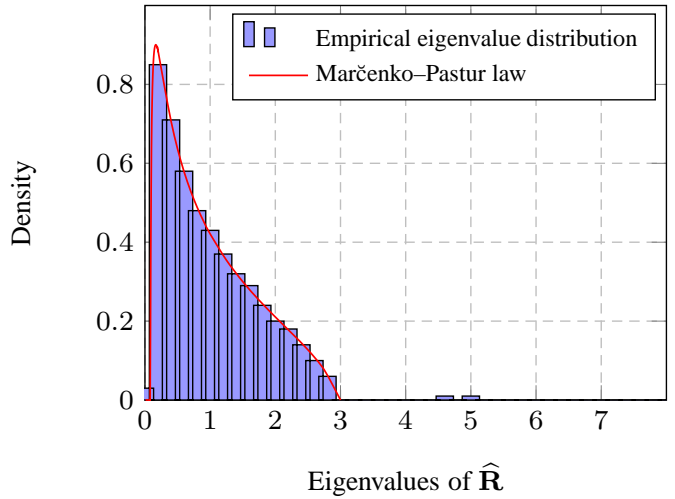

Fig. 1. Histogram of the empirical eigenvalues of $\widehat{\mathbf{R}}$ and the Marčenko-Pastur law for $M=500, \tau=1000, c=0.5, K=2$, $P_{1}=P_{2}=1 / M, I=0$, and $\sigma^{2}=1$.

sponding to the signal part. The main result of this paper provides a separability condition and is presented in the next section. The proof is based on the asymptotic spectrum analysis of $\frac{1}{\tau} \mathbf{Z Z}^{\mathrm{H}}$ and the main steps will be given in Section 4.

\section{MAIN RESULT}

Recall the interference-plus-noise matrix $\mathbf{Z}=\mathbf{H}_{I} \mathbf{X}_{I}+\mathbf{W}$. Let $G$ be the limiting spectral distribution (1.s.d.) of $\frac{1}{\tau} \mathbf{Z Z}^{\mathrm{H}}$ with support denoted by $\operatorname{supp}(G)$ and with the Stieltjes transform ${ }^{4}$ (ST) $m$ (see Theorem 3 for its characterization). Define the interference covariance matrix by $\boldsymbol{\Sigma}_{I} \triangleq \frac{1}{\tau} \mathbf{H}_{I} \mathbf{X}_{I} \mathbf{X}_{I}^{\mathrm{H}} \mathbf{H}_{I}^{\mathrm{H}}$ with l.s.d. $F$ whose ST is denoted by $m_{F}$ and whose support is the interval $\left[x_{1}, x_{2}\right]$ (see Theorem 2). As $m_{F}(z)$ for $z \in \mathbb{C}^{+} \triangleq\{z \in \mathbb{C}, \Im z>0\}$ admits an analytic continuation on $\left(x_{2}, \infty\right)$, we can define $m_{F_{2}} \triangleq$ $\lim _{x \rightarrow x_{2}^{+}} m_{F}(x)$. The following theorem provides the condition for separability and the limiting behavior of the $K$ largest eigenvalues of $\widehat{\mathbf{R}}$.

Theorem 1. Let b be the upper bound of $\operatorname{supp}(G)$. It is given by

$$
b=\frac{m_{F}^{-1}\left(\frac{1}{c \sigma^{2}}\left(1-\frac{1}{1+\sigma^{2} c m_{b}}\right)\right)}{\left(1+\sigma^{2} c m_{b}\right)^{2}}+\frac{\sigma^{2}(1-c)}{1+\sigma^{2} c m_{b}}
$$

where $m_{b}$ is the unique solution in $\left(m_{F_{2}}\left(1-\sigma^{2} c m_{F_{2}}\right)^{-1}, 0\right)$ of

$$
m=\frac{1}{2 \sigma^{4} c^{2} x(m)\left(1+\sigma^{2} c m\right)^{2} m_{F}^{\prime}(\xi(m))}+\frac{1-c}{2 c x(m)}-\frac{1}{\sigma^{2} c}
$$

with

$$
\begin{gathered}
x(m)=\frac{m_{F}^{-1}\left(\frac{1}{c \sigma^{2}}\left(1-\frac{1}{1+\sigma^{2} c m}\right)\right)}{\left(1+\sigma^{2} c m\right)^{2}}+\frac{\sigma^{2}(1-c)}{1+\sigma^{2} c m}, \\
\xi(m)=\left(1+\sigma^{2} c m\right)\left(\left(1+\sigma^{2} c m\right) x(m)-\sigma^{2}(1-c)\right),
\end{gathered}
$$

and

$$
m_{F}^{\prime}(\xi(m))=\int \frac{d F(t)}{(t-\xi(m))^{2}} .
$$

\footnotetext{
${ }^{4}$ The ST $m_{F}$ of a spectral distribution $F$ with support in $\mathbb{R}$ denoted by $\operatorname{supp}(F)$ is defined by $m_{F}(z)=\int(t-z)^{-1} d F(t)$. It is analytic on $\mathbb{C}-\operatorname{supp}(F)$ and completely characterizes the spectral distribution $F$.
} 
Let $m(x)$ be the analytic continuation of $m(z)$ on $(b, \infty)$. Define $\zeta(x) \triangleq M m(x)(\operatorname{cxm}(x)+c-1)$ decreasing from $\zeta\left(b^{+}\right)$to zero on $(b, \infty)$. Let

$$
P_{\lim } \triangleq \frac{1}{M m_{b}\left(c b m_{b}+c-1\right)}
$$

Let $k_{0} \in\{0, \ldots, K\}$ be the largest integer for which $P_{k_{0}}>P_{\mathrm{lim}}$. Let $\lambda_{1} \geq \cdots \geq \lambda_{K}$ be the $K$ largest eigenvalues of $\widehat{\mathbf{R}}$. If $k_{0}=0$, then $\lambda_{1} \underset{M \rightarrow \infty}{\stackrel{a . s .}{\longrightarrow}} b$. Otherwise, for $k=1, \ldots, k_{0}$, let $\rho_{k}$ be the unique solution in $(b, \infty)$ of the equation $P_{k} \zeta(x)=1$. Then,

$$
\lambda_{1} \underset{M \rightarrow \infty}{\stackrel{a . s .}{\longrightarrow}} \rho_{1}, \ldots, \lambda_{k_{0}} \underset{M \rightarrow \infty}{\stackrel{\text { a.s. }}{\longrightarrow}} \rho_{k_{0}} \text {, and } \lambda_{k_{0}+1} \underset{M \rightarrow \infty}{\stackrel{\text { a.s. }}{\longrightarrow}} \text { b. }
$$

From this theorem, if $k_{0}$ among the $K$ signals satisfies the separability condition having their powers strong enough, then the $k_{0}$ largest eigenvalues of $\widehat{\mathbf{R}}$ will converge to limits located outside the right side of $\operatorname{supp}(G)$. If $k_{0}=K$ then all the signals satisfies the separability condition and the signal part is asymptotically separated from the interference-plus-noise bulk. Note that $P_{\text {lim }}$ in (5) is obtained from an exact characterization of the upper bound $b$, in contrast to the approximate characterization provided in [1]. The proof is based on results of [12] and [13] and the main steps are provided in the next section. Note that the values of $b$ and $m_{b}$ can be calculated numerically by solving first the fixed point equation (4) in order to get $m_{b}$.

One application of Theorem 1 is that the BS of interest can suppress the interference-plus-noise by projecting the received sample matrix $\mathbf{Y}$ onto the eigendirections spanned by the $k_{0}$ largest eigenvalues of $\hat{\mathbf{R}}$. If the users of interest have distinct transmit powers $P_{1}>\ldots>P_{k_{0}}$, then it follows that $\rho_{1}>\ldots>\rho_{k_{0}}$ and thus the BS can asymptotically associate the signal received over each of the largest eigenvalues with its true transmitter. If the transmit powers are the same, or if the asymptotic regime has not been fully approached in practice, pilot signals might be needed to correctly separate the users' signals (as in, e.g., $[1,8]$ ). Notice that despite the separation between interference-plus-noise, the signal eigenvalues contain residual interference and noise and $\rho_{k}>M P_{k}$; more precisely, the remaining SNR is $M P_{k} /\left(\rho_{k}-M P_{k}\right)$.

\section{MAIN STEPS OF THE PROOF OF THEOREM 1}

The power limit (5) is obtained from an application of the results of [12]. The main concern of the proof of Theorem 1 is the characterization of $b$ and $m_{b}$. We start by presenting a result [14] on the limiting probability density function of the eigenvalues of $\boldsymbol{\Sigma}_{I}$ and the ST of the corresponding 1.s.d.

Theorem 2 ([14]). Let $\boldsymbol{\Sigma}_{I}=\frac{1}{\tau} \mathbf{H}_{I} \mathbf{X}_{I} \mathbf{X}_{I}^{\mathrm{H}} \mathbf{H}_{I}^{\mathrm{H}}$ where $\mathbf{H}_{I}$ and $\mathbf{X}_{I}$ are defined as in (2). Let $M \rightarrow \infty, M / \tau \rightarrow c \in(0, \infty)$ and $M /(L K) \rightarrow \tilde{c} \in(0, \infty)$. The l.s.d. of $\boldsymbol{\Sigma}_{I}$ is given by $F$ with probability density composed of a point mass at zero that is only vanishing for $\max \{\tilde{c}, c\}>1$ and a continuous part with a compact support. The continuous part $f(x)=c(x) / \pi$ where $c(x)$ is the unique positive solution to

$$
c^{2}(x)=3 g^{2}(x)-2 \frac{c+\tilde{c}-2 c \tilde{c}}{c \tilde{c}} g(x)+\frac{(c-1)(\tilde{c}-1)-x}{c \tilde{c} x^{2}}
$$

for $x_{1}<x<x_{2}$ and 0 elsewhere and the function $g(x)$ represents the unique real solution on $\left[x_{1}, x_{2}\right]$ of

$$
\begin{aligned}
& 8 c \tilde{c} \xi^{2} x^{2} g^{3}(x)-8 x(c+\tilde{c}-c \tilde{c}) \xi g^{2}(x) \\
& +2\left((c-1)(\tilde{c}-1)-\xi x+\frac{(c+\tilde{c}-2 c \tilde{c})^{2}}{c \tilde{c}}\right) g(x) \\
& +\frac{(\xi x-(c-1)(\tilde{c}-1))(c+\tilde{c}-2 c \tilde{c})}{c \tilde{c} \xi x}+1=0
\end{aligned}
$$

with $\xi=1 /(L K I)$ and $x_{1}$ and $x_{2}$ are two largest nonnegative solutions of the equation

$$
\begin{aligned}
& 4 c \tilde{c} \xi^{3} x^{3}-\xi^{2}\left(10 c^{2} \tilde{c}+10 c \tilde{c}+10 c \tilde{c}^{2}-c^{2}-c^{2} \tilde{c}^{2}\right. \\
& \left.-\tilde{c}^{2}\right) x^{2}+2 \xi\left(4 c^{3} \tilde{c}+4 c \tilde{c}+4 c \tilde{c}^{3}-2 c^{2} \tilde{c}-2 c^{2} \tilde{c}^{2}\right. \\
& \left.-2 c \tilde{c}^{2}-c^{3} \tilde{c}^{2}-c^{2} \tilde{c}^{3}-c^{3}-\tilde{c}^{3}-c^{2}-\tilde{c}^{2}\right) x \\
& +(c-1)^{2}(\tilde{c}-1)^{2}(c-\tilde{c})^{2}=0 .
\end{aligned}
$$

Moreover, the ST of $F$, denoted by $m_{F}$, for $x \in \mathbb{R}-\left[x_{1}, x_{2}\right]$, satisfies the equation ${ }^{5}$

$$
\begin{aligned}
& \frac{x^{2} c \tilde{c}}{\xi} m_{F}^{3}(x)-\frac{x(c+\tilde{c}-2 c \tilde{c})}{\xi} m_{F}^{2}(x) \\
& +\left(\frac{(c-1)(\tilde{c}-1)}{\xi}-x\right) m_{F}(x)-1=0 .
\end{aligned}
$$

Making use of this result, the asymptotic spectrum of the interference-plus-noise part can be now characterized.

The following result on the 1.s.d. of the interference-plus-noise covariance matrix is a direct application of [15] (Item 1) and of [16] (Item 2).

Theorem $\mathbf{3}$ ([15], [16]). Let $\mathbf{Z}=\mathbf{H}_{I} \mathbf{X}_{I}+\mathbf{W}$ with $\mathbf{H}_{I}, \mathbf{X}_{I}$, and $\mathbf{W}$ defined as in (2). Let $F$ be the l.s.d. of $\boldsymbol{\Sigma}_{I}=\frac{1}{\tau} \mathbf{H}_{I} \mathbf{X}_{I} \mathbf{X}_{I}^{\mathrm{H}} \mathbf{H}_{I}^{\mathrm{H}}$ defined as in Theorem 2. Define

$$
\boldsymbol{\Sigma} \triangleq \frac{1}{\tau}\left(\mathbf{H}_{I} \mathbf{X}_{I}+\mathbf{W}\right)\left(\mathbf{H}_{I} \mathbf{X}_{I}+\mathbf{W}\right)^{\mathrm{H}} .
$$

Then:

1. As $M \rightarrow \infty$, the empirical distribution function of the eigenvalues of $\boldsymbol{\Sigma}$ converges weakly and a.s. to a non-random distribution function $G$ whose $S T m(z)$, for $z \in \mathbb{C}^{+}$, is the unique solution of

$$
m(z)=\int \frac{d F(t)}{\frac{t}{1+\sigma^{2} c m(z)}-\left(1+\sigma^{2} c m(z)\right) z+\sigma^{2}(1-c)}
$$

such that $m(z) \in \mathbb{C}^{+}$and $z m(z) \in \mathbb{C}^{+}$.

2. Recall $\operatorname{supp}(G)$ is the support of $G$. For any interval $\left[d_{1}, d_{2}\right] \subset \mathbb{R}-\operatorname{supp}(G)$, there exist $M_{0}$ such that for all $M \geq M_{0}$,

$$
\mathbb{P}\left\{\text { no eigenvalue of } \boldsymbol{\Sigma} \text { appear in }\left[d_{1}, d_{2}\right]\right\}=1 .
$$

Note that as $m(z), z \in \mathbb{C}^{+}$admits an analytic continuation on $(b, \infty)$, we have $m_{b}=\lim _{x \rightarrow b}+m(x)$. Notice that depending on the system parameters, $\operatorname{supp}(G)$ may be asymptotically composed of one (the interference spectrum is mixed with the noise spectrum) or two intervals (the interference spectrum is separated from the noise spectrum). The result of Item 2 means that asymptotically no

\footnotetext{
${ }^{5}$ The sign difference in (6) as compared to that in [14] is due to the different definition of the ST used in [14].
} 
eigenvalues due to the interference-plus-noise part can appear outside of $\operatorname{supp}(G)$ with probability one. Hence, if isolated eigenvalues of $\widehat{\mathbf{R}}$ are observed on the right side of $\operatorname{supp}(G)$, they are only due to the signal part.

The final part of the proof consists in derivation of Equations (3) and (4) by using the results of Theorem 2 and Theorem 3. We skip the details of the derivation which is mainly based on the asymptotic support analysis performed in [15].

\section{NUMERICAL RESULTS}

In this section we provide simulation results that compare the separability regions of the proposed method with the results of [1]. All the matrix entries of the model (2) are assumed to have circularly symmetric Gaussian entries. The signal powers are assumed to be all equal $P_{1}=\ldots=P_{K}=P$. Two cases with different noise levels are considered. First, in Figure $2, \sigma^{2}$ is equal to $-10 \mathrm{~dB}$. The ratio $K / M$ is plotted versus the interference-to-signal power ratio $I / P$. The signal is separated from the interference-plus-noise within the region delimited by the horizontal axis, the vertical axis, and the curves. In this scenario, we observe that the new separability region is wider than that of [1]. This means that the result of [1] underestimates the size of the separability region and the signal can be still separated from the interference-plus-noise within the region between the two curves. This is confirmed empirically by the histogram (obtained from 1000 realizations) of the sample covariance matrix in Figure 3 by taking $I / P=0.2$ and $K / M=2 \cdot 10^{-3}$ (the point marked by a square in Figure 2).

The second case considers a higher noise level for which $\sigma^{2}$ is equal to $6 \mathrm{~dB}$ and the curves are plotted in Figure 4. The new bound is tighter than that of [1] meaning that [1] incorrectly predicts separation between the signal and the interference-plus-noise bulk which does not happen in practice. This is confirmed in Figure 5 by taking $I / P=0.4$ and $K / M=2 \cdot 10^{-3}$ (marked by a square in Figure 4).

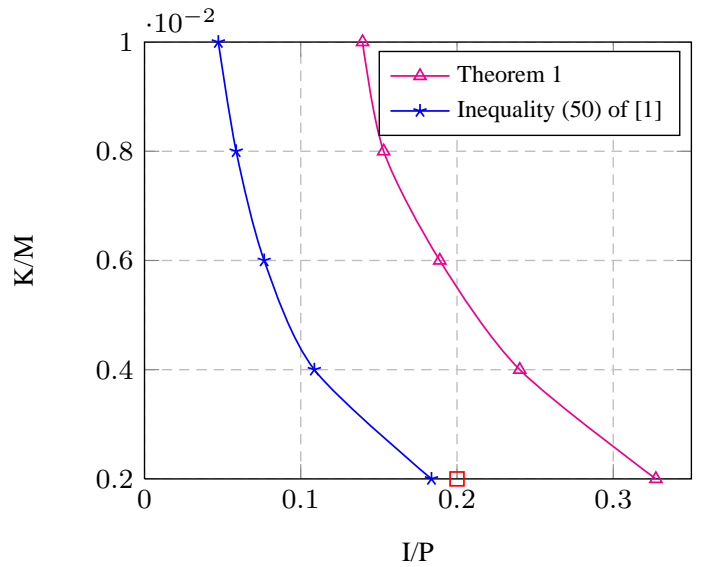

Fig. 2. Separability region for $I=-24 \mathrm{~dB}, \sigma^{2}=-10 \mathrm{~dB}, L=$ $500, \tau=1000$, and $M=1000$.

\section{CONCLUSIONS}

This paper analyzes asymptotic bounds on separability of desired signals and interference-plus-noise from the sample covariance matrix in massive MIMO systems. Our results showed that the bounds provided in [1] can be inconsistent at low SNR and for $L$ large which is due to the approximations used in [1]. In contrast, the proposed bounds are derived by using the exact asymptotic spectrum analysis making it applicable for a wider range of practical situations.

As future work, we will consider an extension to the scenario where a relative attenuation between the out-of-cell users and the $\mathrm{BS}$ of interest is taken into account. In this case, the columns of the channel matrix $\mathbf{H}_{I}$ have different variances.

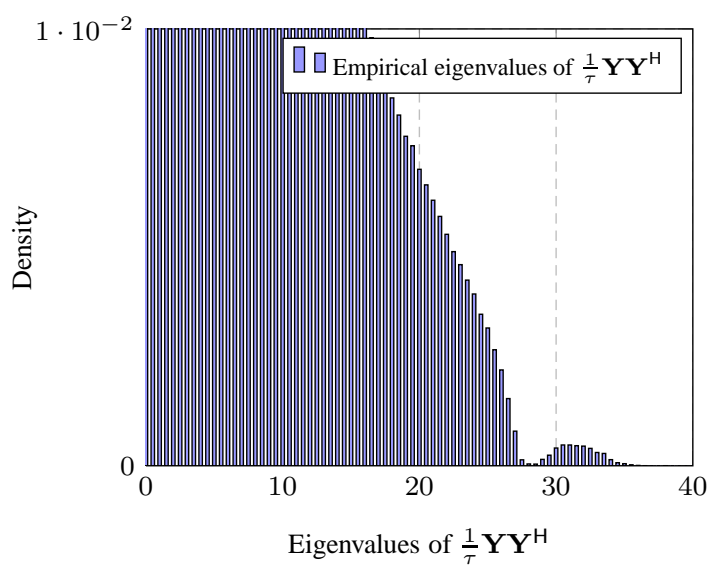

Fig. 3. Empirical eigenvalues of $\frac{1}{\tau} \mathbf{Y} \mathbf{Y}^{\mathrm{H}}$ for $\sigma^{2}=-10 \mathrm{~dB}, I / P=$ $0.2, K=2, L=500, \tau=1000$, and $M=1000$.

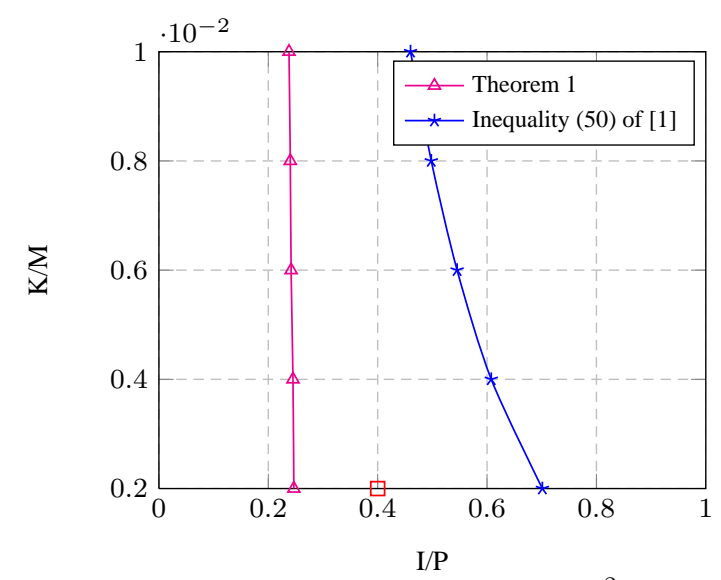

Fig. 4. Separability region for $I=-24 \mathrm{~dB}, \sigma^{2}=6 \mathrm{~dB}, L=500$, $\tau=1000$, and $M=1000$.

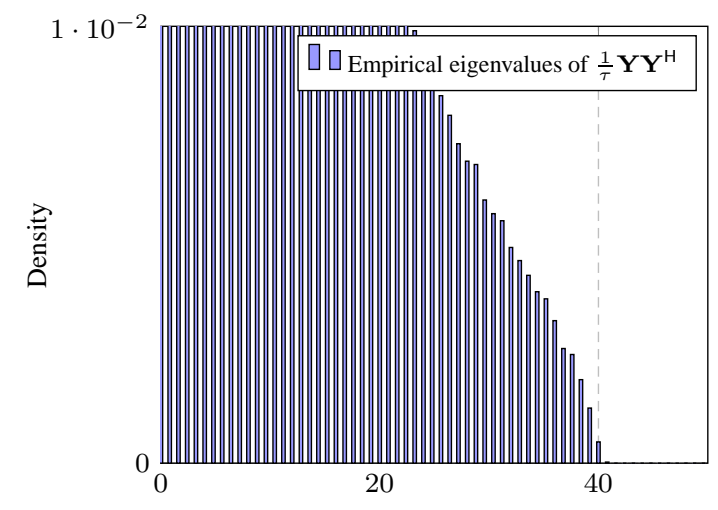

Eigenvalues of $\frac{1}{\tau} \mathbf{Y} \mathbf{Y}^{\mathrm{H}}$

Fig. 5. Empirical eigenvalues of $\frac{1}{\tau} \mathbf{Y} \mathbf{Y}^{\mathrm{H}}$ for $\sigma^{2}=6 \mathrm{~dB}, I / P=0.4$, $K=2, L=500, \tau=1000$, and $M=1000$. 


\section{REFERENCES}

[1] R. R. Müller, L. Cottatellucci, and M. Vehkaperä, "Blind pilot decontamination," IEEE Journal of Selected Topics in Signal Processing, vol. 8, no. 5, pp. 773-786, 2014.

[2] T. L. Marzetta, "Noncooperative cellular wireless with unlimited numbers of base station antennas," IEEE Transactions on Wireless Communications, vol. 9, no. 11, pp. 3590-3600, 2010.

[3] J. Jose, A. Ashikhmin, T. L. Marzetta, and S. Vishwanath, "Pilot contamination and precoding in multi-cell TDD systems," IEEE Transactions on Wireless Communications, vol. 10, no. 8, pp. 2640-2651, 2011.

[4] H. Huh, G. Caire, H. Papadopoulos, and S. Ramprashad, "Achieving "massive MIMO" spectral efficiency with a notso-large number of antennas," IEEE Transactions on Wireless Communications, vol. 11, no. 9, pp. 3226-3239, 2012.

[5] H. Yin, D. Gesbert, M. Filippou, and Y. Liu, "A coordinated approach to channel estimation in large-scale multiple-antenna systems," IEEE Journal on Selected Areas in Communications, vol. 31, no. 2, pp. 264-273, 2013.

[6] E. Björnson, E. Larsson, and M. Debbah, "Massive MIMO for maximal spectral efficiency: How many users and pilots should be allocated?," to appear in IEEE Transactions on Wireless Communications. Available: http://arxiv.org/abs/1412.7102.

[7] H. Q. Ngo and E. G. Larsson, "EVD-based channel estimations for multicell multiuser MIMO with very large antenna arrays," in ICASSP, (Kyoto, Japan), 2012.

[8] D. Neumann, M. Joham, and W. Utschick, "Channel estimation in massive MIMO systems." Submitted. [Online] http://arxiv.org/abs/1503.08691.
[9] J. Ma and L. Ping, "Data-aided channel estimation in large antenna systems," IEEE Transactions on Signal Processing, vol. 62, no. 12, pp. 3111-3124, 2014.

[10] J. Baik and J. W. Silverstein, "Eigenvalues of large sample covariance matrices of spiked population models," Journal of Multivariate Analysis, vol. 97, no. 6, pp. 1382-1408, 2006.

[11] V. A. Marčenko and L. A. Pastur, "Distribution of eigenvalues for some sets of random matrices," Mathematics of the USSRSbornik, vol. 1, no. 4, pp. 457-483, 1967.

[12] J. Vinogradova, R. Couillet, and W. Hachem, "Statistical inference in large antenna arrays under unknown noise pattern," IEEE Transactions on Signal Processing, vol. 61, no. 11, pp. 5633-5645, 2013.

[13] F. Chapon, R. Couillet, W. Hachem, and X. Mestre, "The outliers among the singular values of large rectangular random matrices with additive fixed rank deformation," Markov Processes and Related Fields, vol. 20, no. 2, pp. 183-228, 2014.

[14] R. R. Müller, "A random matrix model of communication via antenna arrays," IEEE Transactions on Information Theory, vol. 48, no. 9, pp. 2495-2506, 2002.

[15] R. B. Dozier and J. W. Silverstein, "Analysis of the limiting spectral distribution of large dimensional information-plusnoise type matrices," Journal of Multivariate Analysis, vol. 98, no. 6, pp. 1099-1122, 2007.

[16] Z. D. Bai and J. W. Silverstein, "No eigenvalues outside the support of the limiting spectral distribution of largedimensional sample covariance matrices," The Annals of Probability, vol. 26, no. 1, pp. 316-345, 1998. 